\title{
AN ABUNDANCE ANALYSIS OF THE SILICON CP STAR HD 43819
}

\author{
ZULEMA LOPEZ-GARCIA (1) \\ Observatorio Astronomico "Felix Aguilar", Universidad \\ Nacional de San Juan, San Juan, Argentina
}

SAUL J. ADELMAN

Department of Physics, The Citadel, Charleston, SC 29409 USA

\begin{abstract}
A fine analysis performed using four $4.3 \AA / \mathrm{mm}$ IIaO Mt. Wilson Observatory spectrograms shows that HD 43819 has abundance anomalies similar to those of other Silicon stars. namely it is helium, carbon, magnesium, and sulfur poor while being silicon, titanium, chromium, manganese, iron, strontium, zirconium, and rare earth rich.
\end{abstract}

\section{INTRODUCTION}

HD 43819 ( = HR 2258) was found to be a pecullar object by Cowley (1972). According to her classification it is a B9IIIp Si star with $\mathrm{Cr}$ probably enhanced. Winzer (1974) found it was a single wave photometric varlable with a period of 1.0785 days. Spectrophotometry by Adelman $(1975,1982)$ revealed a definite $\lambda 5200$ feature, a result confirmed by Maitzen (1980), as well as a modest $\lambda 4200$ feature. A probable magnetic field of $-290 \mathrm{G}$ is given for this star in the Catalogue of Magnetic Field Measurements (Didelon 1983).

HD 43819 has sharp lines with $v \sin i=14 \mathrm{~km} / \mathrm{s}$ (Wolff and Preston 1978). This value is similar to $\mathrm{v} \sin 1=11 \mathrm{~km} / \mathrm{s}$ estimated from the full widths of moderately strong metal lines at the continuum level. The $\mathrm{Ca}$ II $\mathrm{K}$ and $\mathrm{H}$ lines exhibit a secondary component during certain photometric phases (Adelman 1985a).

\section{OBSERVATIONAL MATERIAL AND LINE IDENTIFICATIONS}

Four 4.3 A/mm well-widened spectrograms (Ce 21999, Ce 22006. Ce 22013, and Ce 22576), obtained by one of us (S.J.A.) with the coudé spectrograph of the 2.5-m telescope at Mount Wilson Observatory, were used in this study. The region covered is $\lambda \lambda 3759-4622$.

Line identifications were performed by Adelman (1985b). Lines of C II, Mg I, Mg II, Si II, S II, Ca II, TI II, Cr I, Cr II, Mn II, Fe I, Fe II, Sr II, Zr II, Ce II, Ce III, Nd II and Eu II are definitely present in this star; in

(1) Member of the Carrera del Investigador Cientifico, CONICET, Argentina 
addition, He I, Si I, Sc II, Fe III, Ni I, Ni II, Ba II, Pr II, Gd II, and Hg II are the atomic species with lines which also may be present. Equivalent widths and $\mathrm{H} \gamma$ profiles were read directly from the intensity tracings made with the ald of the PDS microdensitometer of Kitt Peak National Observatory.

\section{DETERMINATION OF ATMOSPHERIC PARAMETERS}

The optical region fluxes were compared with the predictions of solar composition LTE line-blanketed model atmospheres calculated with the ATLAS6 code (Adelman 1982). The Balmer Jump region is fit alone by a $11875 \mathrm{~K}, \log \mathrm{g}=4.0$ model and the Paschen continuum alone by a $11000 \mathrm{~K}$, $\log g=4.0$ model. The $H \gamma$ profile was also compared with the predictions of LTE model atmospheres calculated with the ATLAS8 code. A correction for scattered light was applied. The predictions of a Teff $=11875$ $\mathrm{K}, \log g=3.3$ model fit both the spectrophotometry and the $\mathrm{H} \gamma$ profile. Table 1 and Figure 1 show the agreement of the predicted and observed $\mathrm{H} \gamma$ profile which is an average of all the plates and of both wings.

The $\mathrm{H} \gamma$ profile was calculated from the model atmospheres using the program BALMER8. Program WIDTH8 was used to determine the metal abundances. The adopted metal line damping constants were semiclassical approximations except for those of neutral and singly-ionized $\mathrm{Ca}$ - Ni lines whose adopted values were based on the data of Kurucz (1989 private communication), for lines of C II multiplet 6 and $\mathrm{Mg}$ II multiplet 4 where the adopted values for the Stark broadening were based on data of

Table 1. The Hy Proflle of HD 43819

\begin{tabular}{ccc}
\hline \multicolumn{3}{c}{ R } \\
\multicolumn{1}{c}{ R(A) } & HD 43819 & MODEL \\
\hline 0.0 & 0.216 & 0.210 \\
0.5 & 0.343 & 0.340 \\
1.0 & 0.428 & 0.411 \\
1.5 & 0.478 & 0.459 \\
2.0 & 0.515 & 0.504 \\
3.0 & 0.584 & 0.586 \\
4.0 & 0.656 & 0.658 \\
5.0 & 0.718 & 0.719 \\
6.0 & 0.770 & 0.769 \\
8.0 & 0.846 & 0.844 \\
10.0 & 0.891 & 0.892 \\
12.0 & 0.923 & 0.923 \\
14.0 & 0.945 & 0.943 \\
16.0 & 0.962 & 0.967 \\
18.0 & 0.969 & 0.967 \\
20.0 & 0.975 & 0.974 \\
22.0 & 0.979 & 0.979 \\
24.0 & 0.983 & 0.983 \\
28.0 & 0.986 & 0.986 \\
32.0 & 0.993 & 0.988 \\
36.0 & 0.994 & 0.990 \\
40.0 & 0.995 & 0.991 \\
& & \\
\hline
\end{tabular}


Sahal-Brechot (1969), and for SI II where the damping constants are those of Lanz, Dimitrijevic, and Artru (1988).

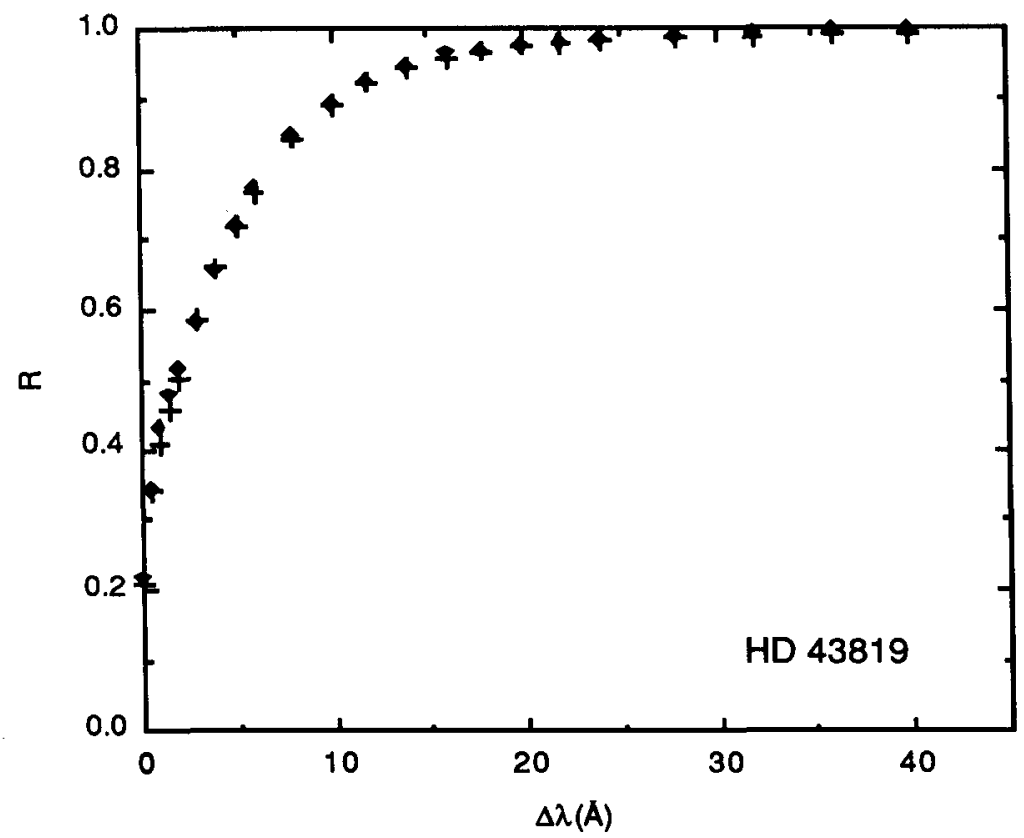

Figure 1. The $\mathrm{H} \gamma$ Profule of $\mathrm{HD} 43819$ with the measurements obtained from the spectrograms (closed diamonds) compared with the predictions of a $11875 \mathrm{~K}, \log \mathrm{g}=3.3$ solar composition line blanketed model atmosphere calculated with the ATLAS8 code (pluses).

Abundances were dertved from Fe I and Fe II lines for a range of possible microturbulent velocities $(\xi)$. For the adopted value (Table 2 ) the derived abundances are not a function of equivalent width. Values for both species were found using lines with of values only from Martin. Fuhr, and Wiese (1988) and also with gf-values from compatible sources, in this case, Kurucz (private communication). The values determined by minimizing the rms scatter of the abundances derived from lines of $\mathrm{Fe} I$ and Fe II agree quite well with those derived by requiring that the

Table 2. Determination of the microturbulent velocity from iron lines

\begin{tabular}{lcccc}
\hline Element & $\begin{array}{c}\text { Number } \\
\text { of lines }\end{array}$ & $\begin{array}{c}\xi \\
(\mathbf{k m} / \mathrm{s})\end{array}$ & $\log \mathrm{Fe} / \mathrm{H}$ & gf-values \\
\hline \multirow{2}{*}{ Fe I } & 52 & 0.80 & $-3.20 \pm 0.32$ & $\mathrm{MF} \& \mathrm{KX}$ \\
& 51 & 0.80 & $-3.20 \pm 0.31$ & $\mathrm{MF}$ \\
Fe II & 70 & 0.80 & $-3.40 \pm 0.34$ & $\mathrm{MF} \& \mathrm{KX}$ \\
& 31 & 0.85 & $-3.44 \pm 0.32$ & $\mathrm{MF}$ \\
& adopted: & 0.80 & & \\
\hline
\end{tabular}


abundances be independent of equivalent width. The microturbulences dertved from other atomic spectes by requiring that the abundances be independent of equivalent width agree also with the results from $\mathrm{Fe} I$ and Fe II (see Table 3).

Table 3. Microturbulent velocities from non-Iron lines

\begin{tabular}{lccc}
\hline Element & $\begin{array}{c}\text { Number } \\
\text { of lines }\end{array}$ & $\begin{array}{c}\xi \\
(\mathbf{k m} / \mathbf{s})\end{array}$ & $\log \mathrm{N} / \mathrm{H}$ \\
\hline Ti II & 45 & 0.70 & $-5.37 \pm 0.30$ \\
CI I I & 28 & 0.75 & $-4.34 \pm 0.30$ \\
\hline
\end{tabular}

The only He I line in the spectral range considered, which might be present, is $\lambda 4026$. Although we have not formally estimated an abundance from it, clearly hellum should be strongly underabundant.

The analysis of the line spectra is available from the authors, with entries for each line giving the multiplet number (Moore 1945), the laboratory wavelength, the logarithm of the gf-value and its source, the equivalent width in $\mathrm{mA}$ and the deduced abundance. Only lines free of blending were considered in the analysis. The sources of gf-values were Biemont et al. (1982), Fuhr, Martin, and Wiese (1988) (MF), Grevesse et al. (1981), Kurucz (1989 prtvate communication)(KX), Lanz and Artru (1985), Martin, Fuhr, and Wiese (1988), Ward (1985), Wiese and Fuhr (1975), Wiese and Martin (1980), Wiese, Smith, and Glennon (1966), Wiese, Smith, and Miles (1969), and Younger et al. (1978).

\section{CONCLUSIONS}

Table 4 compares the derived abundances derlved with solar values from Anders and Grevesse (1989). $n$ the number of lines analyzed. Relative to the Sun, HD 43819 is helium, carbon, magneslum and sulfur poor. It is silicon rich by a factor of 7. All well analyzed elements of the "iron peak" are overabundant: $\mathrm{TI}$ is rich by a factor of $30, \mathrm{Cr}$ by a factor of $50 . \mathrm{Mn}$ by a factor of 40 and $\mathrm{Fe}$ by a factor of 10 . Large abundance anomalles are also found for $\mathrm{Sr}$, overabundant by about a factor of 5000 , and for $\mathrm{Zr}$, overabundant by a factor of 70 (but its abundance is somewhat uncertain based on only three lines). The rare earths are also very overabundant. These results are similar to those found for other Sillcon and magnetic Cp stars.

\section{REFERENCES}

Adelman, S. J. 1975, ApJ, 195, 397

Adelman, S. J. 1982, A\&AS, 49, 668

Adelman. S. J. 1985a. Inf. Bull. Var. Stars, 2701

Adelman, S. J. 1985b, PASP. 97, 976

Adelman, S. J., and Fuhr, J. R. 1985, A\&A, 152, 434

Anders, E., and Grevesse, N. 1989, Geochim. Cosmochim. Acta, 53, 197

Blemont, E., Karner, C., Meyer, G., Trager, F., and zu Putlitz, G. 1982, A\&A, 107, 166

Cowley, A. 1972, AJ, 77, 750

Didelon, P. 1983, A\&AS, 53, 119 
Fuhr, J. R., Martin, G. A, and Wiese, W. L. 1988. J. Phys. Chem. Ref. Data, 17, Suppl. 4

Grevesse, N., Biemont, E., Hannaford, P., and Lowe, R. E. 1981, Upper

Main Sequence CP Stars, 23rd. Llege Astrophys. Coll., p. 211

Lanz, T., and Artru, M. -C. 1985, Physica Scripta, 32, 115

Lanz, T., Dimitrijevic, M. S., and Artru, M. -C. 1988, A\&A, 192, 249

Magazzu, A., and Cowley, C. R. 1986, ApJ, 308, 254

Maltzen, H. M. 1980, Inf. Bull. Var. Stars, 1735

Martin, G. A., Fuhr, J. R. and Wlese, W. L. 1988, J. Phys. Chem. Ref. Data, 17. Suppl. 3

Moore, C. E. 1945, A "Multiplet Table of Astrophysical Interest". Princeton Untversity Observatory

Sahal-Brechot, S. 1969, A\&A, 2, 322

Ward, L. 1985, MNRAS, 213, 71

Wiese, W. L., and Fuhr, J. R. 1975, J. Phys. Chem. Ref. Data, 4, 263

Wiese, W. L., and Martin, G. A. 1980, NSRDS-NBS 68, Part 2, US Government Printing Office, Washington

Wiese, W. L., Smith, M. W., and Glennon, B. M. 1966, NSRDS-NBS 4, US Government Printing Office, Washington

Wiese, W. L., Smith, M. W., and Miles, B. M. 1969, NSRDS-NBS 22, US Government Printing Office, Washington

Winzer, J. E. 1974, Ph. D. Thesis, University of Toronto

Wolff, S. C., and Preston, G. W. 1978, ApJS, 37, 371

Younger, S. M., Fuhr, J. R. Martin, G. A., and Wiese, W. L. 1978, J. Phys. Chem. Ref. Data, 7, 495

Table 4. Comparison of derived and solar abundances

\begin{tabular}{lrrrr}
\hline Element & $\log$ H/H & n & $\log / \mathbf{H}$ & [N/H] \\
\hline C I I & -3.52 & 3 & -3.31 & -0.21 \\
Mg I & -4.89 & 2 & -4.42 & -0.47 \\
Mg II & -4.60 & 4 & -4.42 & -0.18 \\
Si II & -3.59 & 8 & -4.45 & +0.86 \\
S I I & -5.17 & 3 & -4.79 & -0.38 \\
Sc II & -8.91 & 1 & -8.90 & -0.01 \\
Ti II & -5.48 & 45 & -7.01 & +1.53 \\
Cr I & -3.73 & 2 & -6.33 & +2.60 \\
Cr II & -4.62 & 28 & -6.33 & +1.71 \\
Mn II & -4.95 & 4 & -6.61 & +1.66 \\
Fe I & -3.20 & 52 & -4.33 & +1.13 \\
Fe II & -3.40 & 70 & -4.33 & +0.93 \\
Fe III & -3.65 & 1 & -4.33 & +0.68 \\
Ni I & -6.10 & 1 & -5.75 & -0.35 \\
Sr II & -5.42 & 3 & -9.10 & +3.68 \\
Zr II & -7.62 & 3 & -9.40 & +1.86 \\
Ce II & -6.56 & 8 & -10.45 & +3.89 \\
PI II & -6.55 & 1 & -11.34 & +4.83 \\
Nd II & -7.16 & 1 & -10.50 & +3.34 \\
Eu II & -7.93 & 3 & -11.49 & +3.56 \\
Gd II & -6.96 & 2 & -10.88 & +3.92 \\
& & & & \\
\hline
\end{tabular}

